\title{
CHILE Y JAPÓN DURANTE LOS MIL DÍAS DE ALLENDE, 1970-1973: EL CAMINO LATERAL ${ }^{1}$
}

\author{
Chile and Japan during the thousand Allende's days, 1970-1973: The side road
}

\author{
César Ross*
}

\section{RESUMEN}

La Guerra Fría (1947-1991) estuvo caracterizada por el conocido bipolarismo ideológico EsteOeste, el que tuvo alcance mundial y que abarcó todas las dimensiones de la vida pública y privada. Japón y América Latina quedaron bajo la hegemonía de Estados Unidos y, en consecuencia, sus relaciones estuvieron formalmente supeditadas a las rigideces de este contexto histórico. Sin embargo, las tensiones entre Washington y Tokio y el pragmatismo internacional del Gobierno de Allende (1970-1973), constituyeron las claves de la relación entre Santiago y Tokio, en un modelo caracterizado por la heterodoxia, en un esquema que se apartaba de las reglas generales de la Guerra Fría y que hemos denominado relaciones "laterales".

Palabras clave: Chile, Japón, Allende.

\begin{abstract}
The Cold War (1947-1991) was characterized by the well-known East-West ideological bipolarity, which had global reach and covering all aspects of public and private life. Japan and Latin America came under the hegemony of the United States and, consequently, their relations were formally subject to the rigidities of this historical context. However, tensions between Washington and Tokyo and international pragmatism of the Allende government (1970-1973), were the keys to the relationship between Santiago and Tokyo in a model characterized by heterodoxy, in a scheme that deviated from the general rules of the Cold War and we have called "side" relationships.

Keywords: Chile, Japan, Allende.

\footnotetext{
${ }^{1}$ Este artículo es un resultado parcial del Proyecto No 1110820 del Fondo Nacional de Desarrollo Científico y Tecnológico (FONDECYT), titulado: "Un mundo en crisis: El caso de las relaciones entre Chile y Japón, 1930-1973".

*Instituto de Estudios Avanzados, Universidad de Santiago de Chile. Santiago, Chile. Correo electrónico: cesar.ross@usach.cl Artículo recibido el 28 de abril de 2014. Aceptado el 23 de mayo de 2014.
} 


\section{INTRODUCCIÓN}

En la división del mundo, post Segunda Guerra Mundial, Japón y América Latina (AL) quedaron bajo la hegemonía de EE.UU. y sus relaciones estuvieron supeditadas a este contexto histórico (Ross, 2013). Stallings y Székely (1994) denominaron este esquema de relación entre Japón, EE.UU. y AL como de trilateralidad.

Este artículo se centra en las paradojas de estas relaciones bilaterales durante el Gobierno de la Unidad Popular (UP) (1970-73), y considerando que para la historiografía éstas quedaron sujetas al debate general sobre la Guerra Fría (GF) y a la validez de la tesis de la trilateralidad.

En este escenario, las relaciones entre Chile y Japón se caracterizaron por el desarrollo de lo que he denominado un camino lateral, que consistió en la coexistencia entre la continuidad rutinaria de la actividad diplomática y una activa politica bilateral para reducir la incertidumbre que introducían las transformaciones económico-sociales que se impulsaban desde el Gobierno de Allende y que polarizaban crecientemente la realidad contingente de Chile.

Para Japón, el camino lateral implicaba desoír y, en algunos casos, contradecir a Washington, a cambio de garantizar la provisión de materias primas que eran claves en su proyecto industrial. Para Chile, el camino lateral implicaba sortear las restricciones derivadas de su conflicto con EE.UU., mediante un vínculo pragmático y distanciado de valores centrales del proyecto histórico de la izquierda chilena de entonces, a cambio de recursos económicos que permitían financiar las transformaciones revolucionarias que planteaba el Programa de Gobierno (PG) de Salvador Allende.

Este trabajo evidencia, precisamente esta continuidad rutinaria, así como las acciones que el Gobierno de Allende realizó para reducir la incertidumbre, y cómo esto permitió un incremento de la presencia de las empresas privadas de Japón en Chile, las que finalmente fueron las principales aliadas de la UP, pese a la política económica antiimperialista del Gobierno chileno.

Así, el estudio de la política exterior de los mil días de Allende, que realiza este artículo, ayuda a explicar que la continuidad del modelo económico de ambos gobiernos (ideológicamente opuestos), fue parte de un patrón y de una tradición estratégica de Estado que logró su trascendencia. De esta forma, se evidencia que el pragmatismo ideológico superó el pluralismo ideológico, por lo que este último concepto debe ser, por lo menos, relativizado.

Desde el punto de vista de la metodología y las fuentes, este trabajo ha puesto énfasis en la metodología cualitativa, principalmente análisis documental, el que se aplicó a una amplia bibliografía y a un gran número de documentos de archivos inexplorados.

En cuanto a los resultados, y si bien este trabajo partió desde el debate precedente, la base empírica que le ha permitido discutir las ideas en circulación, está sustentada en la documentación depositada en el Archivo Histórico del Ministerio de Relaciones Exteriores de Chile (AHMRREE), donde se ha recopilado notas, télex y oficios reservados, los que han provisto de información inédita, mucha de la cual refuta algunas de las afirmaciones que hasta ahora tenían estatus de tesis.

El artículo está organizado en cuatro partes. La primera, está dedicada a situar el proyecto de la UP en el contexto histórico y en los propósitos nacionales. La segunda 
se aboca a la Política Exterior del PG. La tercera a reconstruir y explicar las políticas bilaterales desarrolladas por Chile y Japón. La cuarta se concentró en explicar el impacto del Golpe de Estado chileno en sus relaciones con Japón. El artículo termina con una breve conclusión y con la sección de bibliografía y fuentes.

\section{ALLENDE EN EL PODER: DE LA INCERTIDUMBRE A LA NEGOCIACIÓN NEORREALISTA}

La campańa presidencial de Allende no hizo sino que reforzar la imagen que se tenía de él (Álvarez, 2010; Harmer, 2011; Sánchez, 2003): un político comprometido con la democracia y con el socialismo. En esta época, tal combinación tenía una sola interpretación (Brands, 2010; Fermandois, 2013; Harmer, 2011; Ulianova, 2009), especialmente para quienes estaban del lado del capital internacional y conscientes del PG de la UP²: Allende se constituiría en una amenaza para sus intereses.

En el ámbito de la política exterior, el PG de la UP se planteó cinco grandes objetivos, cuya concreción implicaba una abierta provocación al orden imperante de la GF en AL. Sin perjuicio de que la escala de Chile no permitía poner ese orden en peligro, el valor simbólico que podía tener un gobierno socialista elegido democráticamente, en un escenario mundial que solo ofrecía modelos similares creados como resultado del uso de la fuerza $^{3}$, era realmente alto. Desde esta perspectiva, el PG de la UP podía llegar a convertirse en un modelo a seguir y esa sí podía ser una amenaza mayor a escala continental.

Aparte de lo estrictamente político, pero asociado a la política exterior, el PG tenía un fuerte carácter económico que se debatía entre objetivos muy ambiciosos y recursos muy escasos (Collier \&Sater, 2004; Meller, 1998). Desde el punto de vista de lo que se llamó en el PG, la construcción de la nueva economía, se plantearon objetivos que tenían efectos directos sobre la política exterior. El principal fue crear un área de propiedad estatal, que debería sustentar el proceso de transformación. Esta área sería formada por las empresas estatales, más aquellas de origen privado que serían expropiadas ${ }^{4}$. En esta línea, se comenzaría con la nacionalización ${ }^{5}$ de las empresas de la gran minería que estaban en poder de capitales extranjeros y de los monopolios nacionales ${ }^{6}$.

Este proceso de nacionalización prefiguró un escenario de conflicto económico local e internacional7 ${ }^{7}$. En el PG de la UP, el Estado no sería un empresario más, sino que el más importante. De paso, el cumplimiento de este plan afectaba los intereses de poderosos enemigos: los propietarios de las empresas transnacionales y el Gobierno de EE.UU., los epítomes del capitalismo de entonces.

\footnotetext{
${ }^{2}$ Programa básico de gobierno de la Unidad Popular (UP, 1969).

${ }^{3}$ Golpes de Estado, guerras civiles, revoluciones, etc.

${ }^{4}$ Expropiación fue el mecanismo mediante el cual, el Estado compraba, de manera forzosa, una empresa al capital privado.

${ }^{5}$ Proceso, apoyado por la expropiación o por otro mecanismo, destinado a reducir la presencia y el porcentaje del capital extranjero, visualizado como expresión del imperialismo económico, dentro de Chile.

${ }^{6}$ De acuerdo al Programa: 1) La gran minería del cobre, salitre, yodo, hierro y carbón mineral; 2) El sistema financiero del país, en especial la banca privada y seguros; 3) El comercio exterior; 4) Las grandes empresas y monopolios de distribución; 5) Los monopolios industriales estratégicos; 6) En general, aquellas actividades que condicionan el desarrollo económico y social del país, tales como la producción y distribución de energía eléctrica; el transporte ferroviario, aéreo y marítimo; las comunicaciones; la producción, refinación y distribución del petróleo y sus derivados, incluido el gas licuado; la siderurgia, el cemento, la petroquímica y química pesada, la celulosa, el papel.

${ }^{7}$ Solo entre 1971 y 1972 los trabajadores de la gran minería (asociados al capital extranjero) hicieron 85 huelgas, tanto inducidos por esas empresas como por sus propios intereses (Collier \& Sater, 2004).
} 
En el discurso del triunfo, pronunciado el 4 de septiembre de 1970 (Allende, 1970), se ratificó el compromiso con el PG de la UP, con sus aliados políticos y con el Pueblo. Para los observadores externos, la suerte estaba echada.

\section{LAPOLÍTICAEXTERIORDEALLENDE: PRAGMATISMOYAPROXIMACIÓN HETERODOXA AL MUNDO}

\subsection{Politica exterior de la UP: Contra el imperialismo politico}

Desde el punto de vista de la política exterior practicada, el Gobierno de la UP tuvo una posición "antiimperialista, antioligárquica y antimonopólica”(Meller, 1998; Sánchez, 2003), lo que le colocaba en conflicto con el área de influencia dominada por Washington y lo que permite relativizar la concepción de "pluralismo ideológico". La UP se alió con los enemigos de la administración Nixon, suscribió y se adhirió a la mayoría de las iniciativas multilaterales que contravenían la posición de éste y desafió directamente el ideario internacional defendido por Washington (Allende, 1978). En este contexto, lo único que podía esperarse era un conflicto creciente, con las obvias consecuencias derivadas de la enorme asimetría que había entre estos países. La excepcionalidad de la experiencia chilena, hizo del país un experimento dentro del laboratorio latinoamericano de la GF. La UP simbolizaba la posibilidad de transformación económico-social. La democracia y el acceso a la participación electoral ya formaban parte del acervo político de Chile. El acceso a los bienes y a una mejor calidad de vida, sin embargo, estaban pendientes. El ideario de la UP, muy inspirado en los enfoques desarrollistas y dependentistas, elaborados por CEPAL (Devés y Ross, 2009), se convirtieron en la pieza clave del pensamiento y del PG de la UP: En lo económico, este proyecto avanzaba respecto de las posiciones más radicales, que ponían en peligro los más caros intereses de la oligarquía nacional y extranjera con presencia en Chile. La amenaza sobre la propiedad, más que sobre la libertad política, fue lo que encendió todas las alarmas y que activó todos los dispositivos tendientes a neutralizar y destruir al Gobierno de la UP.

\subsection{Política económica de la UP: Contra el imperialismo económico}

El proyecto económico de la UP tuvo un impacto decisivo en las relaciones internacionales del país. La conexión causal entre la presencia del capital extranjero, aliado con la oligarquía local y la pobreza del pueblo, constituían la base de la acción económica de este Gobierno (Ramírez, 1965; Sater, 1990). Esta política también tuvo un sesgo pragmático, pero que se veía desdibujado ante la avasalladora imagen de las nacionalizaciones (Sater, 1990).

El logro de este objetivo, colisionaba directamente con los intereses de las empresas de EE.UU., el principal inversionista y socio comercial de Chile (Sater, 1990). Para las empresas homónimas la señal era clara: correrían un destino similar, especialmente si se trataba de países menos poderosos que EE.UU. Sin embargo, no todas abandonaron Chile. 


\subsection{Discurso ideológico y política exterior pragmática:}

Pese a las características del enfoque económico de la UP, Allende estuvo dispuesto a flexibilizar su discurso (Fermandois, 2013) y su acción internacional, practicando intensamente la diplomacia económica. Ésta, realizada desde el primer día del Gobierno de Allende, forma parte de una tradición más antigua (Wilhelmy, 1976). Así, más que "pluralismo ideológico", lo que puede evidenciar es "pragmatismo puro".

Esta práctica no puede encontrarse en los discursos y documentos oficiales, cargados de la épica y retórica revolucionaria, pero sí en los archivos históricos que muestran un Gobierno dispuesto a condecorar a Presidentes y Directores de empresas transnacionales y a usar a sus diplomáticos como colaboradores directos de los intereses de esas compañías, con el propósito de facilitar la llegada y/o permanencia de sus capitales en Chile, en una heterodoxia revolucionaria disociada del discurso oficial.

Esto, que contradice la imagen que se tiene hasta el presente del gobierno de Allende, no es visible en la relación de Chile con las potencias hegemónicas tradicionales, pero sí es constatable en la relación de Chile con las economías emergentes de entonces, como fue el caso Japón.

\section{JAPÓN Y CHILE, LA PARADOJA DE LA GF EN AL: EL CAMINO LATERAL}

Las relaciones entre Chile y Japón se caracterizaron por el desarrollo de un camino lateral, que describe la coexistencia entre la continuidad rutinaria de la actividad diplomática y una activa política bilateral para reducir la incertidumbre, que introducían las transformaciones económico-sociales que impulsaba el Gobierno de Allende y que polarizaban crecientemente la realidad contingente de Chile.

Para Japón, el camino lateral implicaba desoír y hasta contradecir a Washington, a cambio de garantizar la provisión de materias primas que eran claves en su proyecto industrial. Para Chile, implicaba sortear las restricciones derivadas de su conflicto con EE.UU., mediante un vínculo pragmático y distanciado de valores centrales del proyecto histórico de la izquierda chilena de entonces, a cambio de recursos económicos que permitían financiar las transformaciones revolucionarias que planteaba el PG.

Así, el pragmatismo internacional del Gobierno de Allende, y las divergencias entre Washington y Tokio, constituyeron los elementos clave del contexto en que se desarrolló la relación entre Santiago y Tokio, en el esquema que hemos denominado camino lateral.

En perspectiva, las relaciones con Japón se caracterizaron por la coexistencia entre la mantención de la continuidad rutinaria de la actividad diplomática y una política bilateral activa para reducir la incertidumbre que introducía en estas relaciones, las transformaciones económico-sociales que se impulsaban desde el Gobierno de Allende.

\subsection{POLÍTICA JAPONESA: El Pragmatismo Empresarial del Camino Lateral}

Manteniendo una nerviosa normalidad diplomática y mediante una activa acción destinada a reducir la incertidumbre, ambas estuvieron dirigidas a preservar los beneficios de su relación económica. 
4.1.1. Respecto de la continuidad rutinaria de la actividad diplomática, observamos como en 1971, Japón inició una política para solicitar apoyo para la elección de sus candidatos a cargos internacionales, comenzando con la candidatura del Embajador Senjin Tsuruoka ${ }^{8}$ y siguiendo por otras cuatro solicitudes solo en este ańo ${ }^{9}$, en una práctica que se volvió rutinaria ${ }^{10}$.

Otro indicador de la continuidad fue la política de cooperación, como la de introducir el salmón, las numerosas becas para que funcionarios chilenos ${ }^{11} \mathrm{y}$ las visitas para incrementar los negocios bilaterales ${ }^{12}$.

4.1.2. En cuanto a la política para reducir la incertidumbre, se centró en cuatro dimensiones específicas, las que reflejan los argumentos presentados hasta aquí: Primero, los efectos del contexto de las relaciones Tokio-Washington en la política japonesa hacia Chile; segundo, las iniciativas para despejar el camino de los negocios bilaterales; tercero, la Misión Doko, clave para destrabar lo que impedía el desarrollo de la relación con Japón; y cuarto, la información de prensa que incrementó la incertidumbre.

En cuanto a contexto, y dentro de esta política compleja, la Gaimusho (Cancillería japonesa) desplegó una serie de acciones en el sentido opuesto de lo que estaba buscando desde Washington, sobre todo en lo referido a viabilizar la supervivencia económica de Chile. En este tipo de política puede apreciarse con mayor nitidez el camino lateral de esta relación: De Chile no debía extrañarse (Sánchez, 2003); más allá de la tensión bilateral detectada (Michael Schaller, 2010), la conducta esperada de Japón era exactamente la opuesta.

La tensión Washington-Tokio procuró ser zanjada en la reunión que Nixon y Sato sostuvieron en San Clemente, California, el 13 de enero de $1972^{13}$. Según lo informado por el Embajador Pinochet, esta cumbre revivió la división japonesa histórica: una posición, afirmaba que era necesario reforzar la alianza estratégica de cooperación; la otra, sostenía que era preciso "liberarse del tutelaje norteamericano" 14 dando más espacio a relaciones "disidentes" de Japón, como las que tenía con el Gobierno de la UP. Finalmente se impuso la primera.

Por su parte, las iniciativas para despejar los negocios bilaterales se concentraron en el comienzo del Gobierno de la UP. La preocupación del empresariado japonés por el

\footnotetext{
${ }^{8}$ Nota (N) de la Embajada de Japón en Santiago No 10003, del 12 de enero de 1971. AHMRREE.

9 También la candidatura del Dr. Koichiro Takahashi, para miembro Comité Ejecutivo de la Agencia Meteorológica del Japón (30/03/1971); Gobierno de Japón, para ser reelegido como miembro del Consejo de la Organización de Aviación Civil Internacional (16/04/1971); Gobierno de Japón como miembro del Consejo Ejecutivo de la Comisión Oceanográfica Intergubernamental, a la que Chile postulaba simultáneamente y para lo cual coordinaron apoyos mutuos (26/10/1971); Gobierno de Japón como miembro del Consejo Económico y Social de ONU (11/11/1971).

${ }^{10}$ Oficio Ordinario (OO) RI-DGE 95 (29-10-71), AHMRREE.

${ }^{11}$ En N, 10042, 13/04/1971, AHMRREE: se concedió al Gobierno chileno becas para un total de 19 cursos.

${ }^{12}$ Entre otras, cuyo registro se ha encontrado en AHMRREE: Mitsubishi solicita información para participar en la licitación de Ventana II (OC, 540/227, 28/09/1972); Presentación grupo económico japonés (Shin Nihon Shogi Kabushiki Kaisha) para hacer negocios en Chile (Carta presentada a la Embajada chilena en Tokio, 11/01/1973); Interés de compañía japonesa por electrificar ferrocarril de Arica a la Paz (OC, DGE 30/2, 17/01/1973); Misión financiera japonesa visita Chile (OC, DGE 32/13, 18/01/1973); Misión pesquera japonesa visita Chile (OC, DPC 3628, 02/03/1973); Presentación de Nissan Motors para instalarse en Chile, lo que se materializó mediante la visita de Director Exportación a América Latina, Sr. Akahane, y entrevista con Pedro Vuskovic, Vicepresidente de CORFO (OC, DPC 0191/3, 25/01/1973). Todos los documentos AHMRREE.

${ }^{13}$ Doc. 176, 13/01/1972 (informado en 1973)

${ }^{14}$ Doc. 176, 13/01/1972 (informado en 1973)
} 
futuro de sus negocios en Chile ${ }^{15}$ estimuló la entrevista del Embajador Sakito Sato con el Canciller Almeyda y el Presidente Allende en diciembre de $1970^{16}$ y la instalación de una oficina de la Japan External Trade Organization (JETRO) ${ }^{17}$ en Chile ${ }^{18}$.

En cuanto a la Misión Doko, ella visitó Chile entre el 5 y 8 de diciembre de $1971^{19}$, con el objeto de diagnosticar las reformas de la UP y sus efectos en la economía.

Según el Informe de la Misión presidida por Toshio Doko ${ }^{20}$, se entrevistaron con Salvador Allende, Presidente; Clodomiro Almeyda, Canciller; Pascual Barraza, Ministro de Obras Públicas y Transporte; Orlando Cantuarias, Ministro de Minería; Gonzalo Martner G., Director de la Agencia de Planificación Económica; Barberiz, Jefe de la Comisión Nacional de Investigación Científica y Tecnológica (CONICYT); Alfonso Inostroza, Presidente del Banco Central; Patricio Morales, Subsecretario de Hacienda; Kurt Dreckmann, Vice-Presidente de la Corporación de Fomento de la Producción; y Juan Somavía, Presidente de la Comisión del Grupo Andino. Adicionalmente, se conversó con representantes del sector privado y algunos miembros de la Misión hicieron visitas a la Provincia de Concepción (para ver la Acería de Huachipato y el Puerto de Talcahuano) y a la Mina de Río Blanco. La Misión Doko se centró en la situación de la economía chilena, la posición de Chile y en formarse una visión propia.

Respecto de la economía chilena, la Misión planteó tres cuestiones: Primero, que las medidas que se habían implementado eran mucho más moderadas de lo que se pensaba inicialmente y que casi todas ellas estaban dirigidas a recuperar rápidamente la economía, a reducir la cesantía y a hacer más justa la distribución del ingreso. Segundo, que el Gobierno tenía mucho interés de aumentar en más de $10 \%$ la producción total de cobre y del mineral de hierro. Tercero, que el balance del comercio bilateral era adverso para Japón ${ }^{21}$.

En cuanto a la posición de Chile, se sistematizó las presentaciones del Presidente Allende y de los altos funcionarios con que se habían reunido. Se puso énfasis en explicar la difícil situación chilena, la necesidad de comprensión de parte de Japón, especialmente en lo referido a los créditos internacionales y en las posibilidades que las empresas japonesas hicieran negocios en Chile. Aquí el Ministro de Minería reiteró la posición oficial: a) "la política de nacionalización de las minas de Chile no ha sido bien comprendida. Chile necesita la ayuda extranjera excepto para la gran minería. Para las minas medianas puede ser una empresa mixta -Gobierno 51\%, y capital extranjero $49 \%$ - y en cuanto a las minas pequeñas, no hay problema si el $100 \%$ del capital es extranjero" ${ }^{22}$; b) Chile recibiría "con agrado las inversiones que en el campo de la minería chilena ofreciera el Japón, y cumpliremos todos los compromisos internacionales. [y agregaba entre paréntesis] Si es necesario, es posible hacer un documento oficial diciendo que no se nacionalizará Santa Clara con capitales japoneses"23.

\footnotetext{
${ }_{15}$ OC, ECB 41/2, 21/1/1971 (AHMRREE), del Embajador Augusto Marambio al Ministro de Relaciones Exteriores.

${ }^{16}$ OC, DEAT-RI 496/46, 16/9/1971 (AHMRREE).

${ }^{17}$ JETRO: es el organismo oficial de Japón para el Comercio Exterior.

${ }^{18} \mathrm{~N}, 10051,23 / 4 / 1971$ (AHMRREE).

${ }^{19}$ Junto con Argentina y Brasil.

${ }^{20}$ Anexado al OC, DGE-RI 121/18, 2/3/1972 (AHMRREE).

${ }^{21}$ Anexado al OC, DGE-RI 121/18, 2/3/1972 (AHMRREE).

${ }^{22}$ Anexado al OC, DGE-RI 121/18, 2/3/1972 (AHMRREE).

${ }^{23}$ Anexado al OC, DGE-RI 121/18, 2/3/1972 (AHMRREE).
} 
La Misión concluyó con una convicción optimista que explica la conducta japonesa para el resto del Gobierno de la UP. Básicamente, el Informe apeló a la amistad histórica y a la complementación natural, declarando su visión positiva del futuro, recomendando ofrecer a Chile la colaboración económica que se pueda prestar en cada caso ${ }^{24}$.

Finalmente, las informaciones de prensa, dedicadas a los asuntos internacionales llegaba con efectividad a los tomadores de decisión. Los medios, gradual y crecientemente fueron dando forma a la incertidumbre que había respecto de la situación de Chile, comenzando con la visita de Fidel Castro ${ }^{25}$.

Desde fines de 1971 comenzaron a publicarse titulares preocupantes sobre Chile ${ }^{26}$. En abril de 1972 reaparecieron noticias sobre la difícil situación ${ }^{27}$. En agosto las noticias fueron más explícitas ${ }^{28}$ y en octubre ya eran alarmantes ${ }^{29}$. Según el Informe de 1973 de la Embajada de Chile en Tokio, en julio se publicaron 19 noticias ${ }^{30}$, en agosto $26^{31}$ y en septiembre (solo entre el 1 y 10) $54^{32}$.

Las noticias de septiembre dieron cuenta de una situación cada vez más crítica ${ }^{33}$. La lista comenzó con el titular "Dinamita explota en la capital durante discurso de Allende" (The Japan Times) y terminó con "Las huelgas agotan abastecimientos de alimentos en Chile" (Mainichi).

\subsection{POLÍTICA CHILENA: El Pragmatismo Revolucionario del Camino Lateral}

El vínculo con Tokio tuvo todo el tacto y la flexibilidad que se estimaron necesarias para apalancar los recursos que la revolución chilena requería, así como todos los gestos diplomáticos y los acuerdos económicos, que estos resultados demandaran.

4.2.1 Respecto a la continuidad rutinaria de la actividad diplomática, se procuró reciprocar la conducta iniciada por Japón, al tiempo que se desplegó una intensa política para reforzar la asociatividad económica, lo que Japón condicionó a la regularización de la deuda que el país tenía con la banca nipona.

\footnotetext{
${ }^{24}$ Anexado al OC, DGE-RI 121/18, 2/3/1972 (AHMRREE).

${ }^{25}$ OO, 604/300, 25/11/1971, (AHMRREE).

${ }^{26}$ OO, 632/318, 9/12/1971 (AHMRREE).

${ }^{27}$ OC, RIO 190/79, 6/4/1972 (AHMRREE).

${ }^{28}$ OC, 482/212, 25/8/1972 (AHMRREE). Se elabora una lista de los titulares más destacados: "Terroristas se evaden de prisión, secuestran avión a Chile" (Yomiuri), "Aumenta violencia en Chile. El Ejército asume poderes policiales en Santiago" (The Japan Times), "Estado de Emergencia en Santiago" (Mainichi) y "El Ejército prohíbe las protestas en Provincia de Santiago" (The Japan Times).

${ }^{29}$ Oficio Confidencia 561/234, 13/10/1972 (AHMRREE). Se elabora, como se hacía regularmente, una lista de los titulares más destacados: "Finaliza huelga estudiantil" (Mainichi), "Chile espera embargos de Kennecott" (Mainichi), "Allende advierte al país de futuras privaciones" (The Japan Times), "Chile es refugio para activistas" (The Japan Times), "Chile declara estado parcial de emergencia" (The Japan Times) y "Los chilenos denuncian a Allende" (The Japan Times).

${ }^{30}$ OC, DC 309/138, 5/7/1973 (AHMRREE). Con la lista de 19 noticias publicadas por medios como The Japan Times, Yomiuri y Mainichi, donde destacaron titulares como: "Allende denuncia Golpe del Ejército chileno", "5 chilenos derechistas solicitan asilo en Ecuador" y "Allende planifica formar gabinete totalmente civil".

${ }^{31}$ OC, 348/157, 2/8/1973 (AHMRREE). Se elabora una lista de los titulares más destacados: "Trabajadores chilenos ocupan avenida principal en Santiago" (The Japan Times), "Asesinos del Edecán de Allende eluden a la policía chilena" (Yomiuri), "Explosión daña embajada de Corea del Norte en Santiago" (Yomiuri) y "Chilenos izquierdistas disparan sobre camioneros huelguistas" (The Japan Times).

${ }^{32}$ A contar del 11 de 1973 el número de noticias sobre Chile comenzaría a descender gradualmente.

${ }^{33}$ OC, 411/177, 17/9/1973 con las noticias del lapso 1 a 10 de septiembre de 1973 (AHMRREE).
} 
En este contexto la política chilena fue casi siempre reaccionando a la iniciativa japonesa, en una estrategia de adaptación y avance, que en muchos momentos parecía estar relativamente desconectada de lo que iba ocurriendo en Chile. En parte, era el peso de la tradición diplomática chilena y en parte, era la necesidad de hacer la abstracción necesaria para alcanzar los objetivos planteados para esta relación.

Uno de los primeros gestos ocurrió en marzo de 1971, con el apoyo de Japón para que Santiago fuera sede de la Tercera UNCTAD.

En septiembre de 1971, se solicitó a Japón el apoyo para la candidatura de Felipe Herrera, a la Secretaría General de $\mathrm{ONU}^{34}$. Pese a la insistencia de Santiago ${ }^{35}$, fue electo Kurt Waldheim.

En octubre de 1971, el propio Presidente Allende, envió una carta a Shujiro Fujino, Presidente de Mitsubishi Corporation, invitándolo a él y a una delegación, para visitar Chile y tomar contacto con "personeros de nuestras empresas estatales y de la actividad privada, a fin de analizar el amplio campo de posibilidades que existen en nuestras relaciones futuras" ${ }^{\text {" }}$.

En 1972 Toshio Doko ${ }^{37}$ y Shigeo Nagano ${ }^{38}$ recibieron la condecoración, Bernardo O’Higgins en el grado de Gran Cruz, la distinción más alta entregada por Chile a un extranjero. A la carta de agradecimiento, recibieron otra del propio Presidente Allende ${ }^{39}$. Si en lo formal se trató de la continuidad de una rutina diplomática, ésta estuvo cargada de un excepcional pragmatismo que superaba el mero "pluralismo ideológico".

La acción de continuidad rutinaria más relevante emprendida por el Gobierno de la UP hacia Japón fue la Misión Inostroza, entre el 18 y 23 de marzo de 1972. Presidida por el Presidente del Banco Central, Alfonso Inostroza, tenía como objetivo renegociar la deuda que Chile mantenía con la banca japonesa y que constituía un obstáculo para emprender nuevos negocios, mediados por nuevos créditos nipones. Junto con Inostroza, viajaron Jorge Arrate, Vicepresidente Ejecutivo de la Corporación del Cobre y Sergio Cuevas, Subgerente de Chuquicamata. En Tokio se les unió Jorge Valdovinos, Encargado de Negocios de la Embajada de Chile. Sostuvieron reuniones con representantes del sector privado $^{40}$ y público $^{41}$.

En su argumentación, Inostroza apeló al esquema clásico de la deuda externa (vínculos entre bancos privados, industrias relacionadas y países endeudados) a favor de su argumentación, afirmando que Chile aceptaría este modelo a cambio de la renegociación. En este escenario Inostroza preguntó directamente por el rol que Japón jugaría en el proceso chileno, considerando una serie de antecedentes que indicaban que deberíamos esperar un cierto grado de adhesión ${ }^{42}$. Pese a la esperable prudencia gubernamental, se

\footnotetext{
${ }^{34}$ Télex OIN 76 (Cifrado), 23/9/1971 (AHMRREE).

${ }^{35}$ Nota Verbal (NV) 510/54, 27/9/1971 y NV 550/59, 18/9/1971 (AHMRREE).

${ }^{36}$ Dossier OO, sin codificar, 1971 (AHMRREE).

${ }^{37}$ Presidente de Toshiba Electronic y Vicepresidente de la Federación de Organizaciones Económicas (Keidanren).

${ }_{38}$ Presidente de la Asociación de Amistad Nipo-Latinoamericanas, Presidente de la Cámara de Comercio de Japón, Presidente de Nippon Steel Co. y Representante de Japón en el Consejo Económico de la Cuenca del Pacífico.

${ }^{39}$ OC, GM 576/36, 20/10/1972.

${ }^{40}$ Citadas en la Agenda de la visita: Cía. Komatsu-Bucyrus, Marion International, Grupo Sumitomo, Mitsubishi Corporation, Nissan Motors Co. Ltd., y C. Itoch and Co., Keidanren. OC, DGE-RI 174/20, 30/3/1972 (AHMRREE).

${ }^{41}$ Citadas en la Agenda de la visita: Ministerio de Relaciones Exteriores de Japón (METI), Ministerio de Comercio Exterior e Industria (MITI) y Banco de Japón. OC, DGE-RI 174/20, 30/3/1972 (AHMRREE).

${ }^{42}$ Carta del Presidente Allende al Primer Ministro Sato en 1971 y respuesta de éste, afirmaciones de la Misión Doko y de otros altos funcionarios japoneses.
} 
logró el entusiasmo y compromiso del sector privado, el que siguió llevando adelante iniciativas hacia Chile hasta el final del Gobierno de la UP. A fines de 1972 se alcanzó el principal resultado de la Misión Inostroza: la suscripción del acuerdo de renegociación de la deuda chilena con Japón ${ }^{43}$.

Casi como una anécdota, en esta continuidad rutinaria diplomática, los archivos consignan la solicitud de apoyo para la candidatura de Pablo Neruda, como integrante del Consejo Ejecutivo de UNESCO ${ }^{44}$.

Lo que no puede estimarse una cuestión menor, fue la contratación de un estudio japonés (Braun Morita Hoashi \& Kubota) para que asesora al Estado chileno en la posible demanda y embargo de Kennecott Copper Corporation ${ }^{45}$. Esto significa una sola cosa: Japón no impediría que el gobierno de Chile controlara la gran minería, si ello garantizaba a las empresas japonesas estos recursos a escala de la mediana y pequeña minería ${ }^{46}$.

4.2.2 Respecto a la reducción de la incertidumbre, muchas de las actitudes hacia el empresariado japonés fueron desde ya, acciones en tal dirección. Sin embargo, la política chilena para garantizar el respeto a los intereses japoneses en Chile y el viaje del Canciller Almeyda a Japón, fueron gestos de valor práctico y simbólicos.

La garantía sobre las inversiones japonesas en Chile fue una oferta hecha en el marco de la Misión Doko, por parte del Ministro de Minería, lo que fue discutido por el Embajador de Japón en Santiago, Sakito Sato, con el Canciller de Chile, a dos semanas de concluida la misión japonesa ${ }^{47}$. La reacción del Gobierno de la UP se tradujo en un documento oficial y una estrategia diplomática puesta en marcha en enero de $1971^{48}$.

La estrategia diplomática supuso una serie de entrevistas del entonces Embajador de Chile en Tokio, Augusto Marambio, con agentes estatales y no estatales, a fin de conseguir la confianza en la promesa chilena. Entre muchas otras gestiones, el Embajador Marambio se reunió con el Embajador Hitomi, Director General para los Asuntos de Centro y Sudamérica del Ministerio de Asuntos Extranjeros; con Shigeo Nagano, Presidente de la Cámara de Comercio e Industria de Japón; con Kogoro Uemura, Presidente de la Federación de Organizaciones Económicas de Japón (Keidanren); y con Eiji Kawasaki, ex Embajador de Japón en Chile y Director General de la Sociedad Latinoamericana que, en consultas exploratorias -encomendadas por los círculos económicos japoneses- además visitó en Chile a destacados personeros japoneses de la actividad nacional y al mismo Presidente Allende. Como resultado de esta gestión, el Embajador señor Eiji Kawasaki preparó un informe para ser publicado por la "Revista de Informaciones Económicas Japonesas". Adicionalmente, el Presidente de la República y el Canciller Almeyda, se entrevistaron con el Embajador de Japón en Santiago.

\footnotetext{
${ }^{43}$ Firmado el 28/11/1972 y publicación en el Diario Oficial el 29/11/1972. Aerograma (A) Confidencial Urgente EAT 418/63, del 14/7/1972; A, DGE 705/96, 13/12/1972; OC, OI 526/30, 20/9/1972; y OC, DGE 593/39, 27/10/1972 (todos AHMRREE).

${ }^{44}$ A, OI 493/75, 30/8/192 (AHMRREE), del Embajador Oscar Pinochet al Ministro de Relaciones Exteriores.

${ }^{45}$ Cartas del Estudio dentro de dossier de Oficios Ordinarios de 1972, documentos sin numerar (AHMRREE).

${ }^{46}$ Según informe de 1973, Japón compraba el 35\% de Trióxido de Molibdeno y Ferromolibdeno chilenos, insumos clave en las aleaciones de acero.

${ }^{47}$ Documento La Misión Doko estuvo en Chile hasta el 8 de diciembre y la reunión de Almeyda y Sato fue el 15 de diciembre de 1970.

${ }^{48}$ OC, ECB 41/2, 21/1/1971 (AHMRREE).
} 
El resultado fue la confianza de la parte japonesa. Esta gestión fue una pieza clave del éxito de la relación de Chile con Japón en este período, tan importante como el logro obtenido por la Misión Inostroza al año siguiente.

A comienzos de 1973, el Canciller Almeyda visitó Japón proveniente de una gira que ya había contemplado la República Popular China y a la República Democrática Popular de Corea.

Si bien era observable que el sector privado siempre había tenido más entusiasmo por cooperar con el Gobierno de la UP, en este viaje la brecha entre sector público y privado japonés de hizo más evidente.

El Informe de la Embajada chilena en Tokio fue sutilmente elocuente ${ }^{49}$. Señaló que la visita del 5 y 6 de febrero no era oficial. Indicó que "hubo dificultades en la obtención de las entrevistas con los Ministros Ohira y Aiichi, por cuanto ambos tenían importantes compromisos en las fechas indicadas, razón por la cual las entrevistas fueron de escasa duración y se limitaron a saludos de cortesía" ${ }^{\text {". }}$. Registró la tibia reacción del Director del Ministry of International Trade and Industry (MITI), Minoru Koyama, frente a la iniciativa chilena de enviar una misión de alto nivel para discutir la cooperación bilateral y una nueva renegociación de la deuda chilena, ante lo cual éste habría respondido que se realizarían "los estudios convenientes" 51 . Constató que el vivo interés del sector empresarial distaba mucho del mostrado por el Gobierno japonés, lo que había sido planteado explícitamente por los representantes de las corporaciones empresariales niponas ${ }^{52}$, como el Vicepresidente de Mitsui \& Smelting Co. que buscaba ampliar los negocios en Chile ${ }^{53}$, y que constituía un obstáculo práctico para implementar algunos negocios que requerían la autorización del MITI, incluso en créditos directos concedidos por las empresas, como fue el caso de Komatsu y sus negocios con la Corporación Nacional del Cobre (CODELCO) ${ }^{54}$. Por último, el Presidente de Keidanren, Kogoro Uemura, afirmó que el sector estaba dispuesto a recomendar al Gobierno que actuara con mayor liberalidad frente a Chile.

Los intentos del sector privado japonés continuaron casi hasta el último día del Gobierno de la UP, pero con un apoyo gubernamental cada vez más tenue. Al parecer, las noticias sobre Chile y la diplomacia de Washington eran dos poderosas razones para tomar esta decisión.

\section{El colapso del gobierno de la UP en Chile ${ }^{55}$ :}

Frente a la crisis chilena, ambos países actuaron con un pragmatismo a toda prueba, que preservó la continuidad de la rutina diplomática y que trabajó intensamente

\footnotetext{
${ }^{49}$ OC, DGE-RIO 75/6, 8/2/1973 (AHMRREE)

${ }^{50}$ Ibídem.

${ }^{51}$ Ibíd

${ }^{52}$ Representantes de Kaidanren: Presidente, Kogoro Uemura; Vicepresidente, Toshio Doko; Vicepresidente de Mitsui Mining \& Smelting Co., Orihei Kusano; Vicepresidente y Director Ejecutivo de Ataka and Co., Toshio Misuta; Director Gerente de Komatsu Mfg. Co., Hirosuke Hiroaka; Director Gerente de Nissan Motor Co., Masataka Okuma; Director Gerente de Kaidanren, Rikuzo Koto; y Gerente General de Tokyo Shibaura Electric Co., Haruo Seki. OC, DGE-RIO 75/6, 8/2/1973 (AHMRREE).

${ }^{53}$ OC, DGE-RIO 75/6, 8/2/1973 (AHMRREE).

${ }^{54}$ OC, DGE-RIO 75/6, 8/2/1973 (AHMRREE).

${ }^{55} \mathrm{La}$ base inicial de esta sección proviene de otro artículo elaborado por César Ross, aunque un trabajo posterior de fuentes ha permitido matizar algunas de las ideas presentadas en dicha publicación (Ross, 2005).
} 
para reducir al máximo el impacto que implicaba este nuevo cambio institucional. Así, Japón reconociendo al nuevo Gobierno al día siguiente ${ }^{56}$ del Golpe de Estado.

Entre el 11 y el 20 de septiembre la prensa en inglés editada en Japón, publicó 52 noticias sobre algún aspecto del Golpe de Estado en Chile. Se hablaba de los centenares de muertos, de los miles de arrestados y de los miles que serían enjuiciados, donde se incluía a extranjeros ${ }^{57}$.

En este escenario, y a solo dos semanas del Golpe, la compañía japonesa Toshiba Kasei Kogyo Co. Ltd., se comunicó con la Embajada de Chile en Tokio para ofrecer sus productos, para lo cual envió catálogos cuyo destino sería el sistema de salud chileno ${ }^{58}$.

Aparentemente, se trataba de un momento muy inoportuno para esta gestión, pero no era una excepción para la forma en cómo el empresariado y la política japonesa veían los negocios y la situación de Chile.

\section{CONCLUSIÓN}

Considerando la excepcionalidad ideológica y política del Gobierno de la UP, el examen de las relaciones entre Chile y Japón, en ese período, ha tenido gran valor no solo para contrastar la utilidad de las tesis estándar sobre las relaciones internacionales de Chile en tiempos de GF, sino que para descubrir en la política exterior de Chile un patrón de largo plazo.

El PG de la UP era una promesa de peligro para los intereses de las empresas internacionales que hacían negocios en Chile, especialmente las compañías cuyas casas matrices estaban en EE.UU. En consecuencia, la paradoja internacional derivada del PG no provenía tanto de las implicancias de su supuesto "pluralismo", sino que de su promesa de hacer exactamente lo opuesto, vale decir, de que la brecha entre la política exterior declarada y la política exterior practicada fuera mínima, como en efecto suponían los informes internacionales (diplomáticos y de prensa) que se hacían sobre Chile.

Pese a la expectativa respecto de la política exterior de la UP, alimentada por las afirmaciones vertidas en el PG de la UP y ratificadas por la beligerancia del proceso político chileno, la evidencia documental demuestra que hubo un claro divorcio entre la política exterior declarada y la practicada, con mayor moderación y sobre todo mayor pragmatismo, especialmente en sus relaciones con potencias no hegemónicas, donde la política hacia Japón fue un ejemplo paradigmático.

Las tensiones entre Washington y Tokio y el pragmatismo internacional del Gobierno de Allende, constituyeron los elementos clave del contexto de la relación entre Santiago y Tokio, en un modelo caracterizado por el pragmatismo, en un esquema que se apartaba de las reglas generales de la GF y que en este texto hemos denominado relaciones "laterales".

Las relaciones del Gobierno de la UP con Japón se caracterizaron por la coexistencia entre continuidad rutinaria de la actividad diplomática y una política bilateral activa para reducir la incertidumbre sobre Chile y la reacción japonesa frente a ella.

\footnotetext{
56 "La embajada de Japón se complace en manifestar, por instrucciones de su gobierno al honorable Ministerio de Relaciones Exteriores que el gobierno del Japón desea mantener las mejores relaciones de amistad con el ilustre gobierno de Chile. La Embajada del Japón se vale de la oportunidad para reiterarle al honorable Ministerio de Relaciones Exteriores la seguridad de su más alta y distinguida consideración”. N, 10153, 2/10/1973 (AHMRREE).

${ }^{57}$ OC, 412/178, 20/9/1973 (AHMRREE).

${ }^{58}$ Ibid.
} 
En perspectiva histórica, la política exterior de Chile hacia Japón y hacia el resto del mundo, exhibe enormes similitudes con las características de la política exterior del gobierno anterior (1964-1970) (Wilhelmy, 1976) y con los siguientes. Pese a las diferencias ideológicas entre éstos, se observa la existencia de un patrón que estaría dando cuenta de una continuidad mayor, en lo que podría denominarse como el path dependence de la política exterior de Chile.

Este patrón está dado por la combinación de pragmatismo en la política practicada e idealismo en la política declarada. Se trata de la cohabitación de dos dimensiones que tienden a divergir. Esta afirmación está respaldada por otras investigaciones, con base empírica de información primaria ${ }^{59}$ y solo contradicha por trabajos elaborados en base a información secundaria ${ }^{60}$.

El factor de cambio ha estado ligado a la ideología de cada coalición gobernante. Son evidentes las diferencias valóricas entre unas y otras, especialmente entre la UP y la Dictadura. La trampa interpretativa estuvo precisamente allí, en suponer que la identidad ideológica determinaría la conducta internacional de cada gobierno, en una transferencia automática, pero, como hemos expuesto, no fue así.

El factor de continuidad, que resulta dominante, aparece centrado en la dosis de pragmatismo "realista" en las decisiones, cuyo resultado final ha tendido a ser muy consistente. Dicho de otro modo, casi sin importar el enfoque ideológico y doctrinario del gobierno de turno $^{61}$, la política practicada ha tenido este componente de manera muy patente y consistente.

\section{REFERENCIAS}

Allende, Salvador. Discurso de Victoria Electoral, 1970. Disponible en: http:// www. marxists.org/espanol/allende/05-09-70.htm

Salvador Allende y América Latina: 12 discursos y 2 conferencias de prensa, (1978): 135-163. In C. d. C. e. México (Ed.), Discurso en la Asamblea General de las Naciones Unidas (pp. 135-163). México: DIBAM.

Álvarez, Rolando. "La Unidad Popular y las elecciones presidenciales de 1970 en Chile: La batalla electoral como vía revolucionaria”, Revista del Observatorio Social de América Latina 11/28 (2010): 219-243.

Brands, Hal. Latin America's Cold War. Cambridge, Mass.: Harvard University Press, 2010.

Collier, Simon \& Sater, William F. A history of Chile, 1808-2002. 2a Edition. Cambridge England, New York, N.Y.: Cambridge University Press, 2004.

Devés, Eduardo y Ross, César. Las ciencias económico sociales latinoamericanas en África Sudsahariana. Santiago de Chile: Ariadna Ediciones, 2009.

\footnotetext{
${ }^{59}$ Archivos, como el utilizado para este caso.

${ }^{60}$ Libros y artículos académicos, prensa, entrevistas a testigos de época.

${ }^{61}$ Integracionista latinoamericano (en sus variadas vertientes), neorrealista y neoliberal, entre otros.
} 
Fermandois, Joaquín. La Revolución Inconclusa: La izquierda chilena y el gobierno de la Unidad Popular. Santiago de Chile: Centro de Estudios Públicos, 2013.

Harmer, Tanya. Allende's Chile and the Inter-American Cold War. Chapel Hill: University of North Carolina Press, 2011.

Meller, Patricio. Un siglo de economía politica chilena (1890-1990). Santiago de Chile: Editorial Andrés Bello, 1998.

Ramírez, Hernán. Los Estados Unidos y América Latina (1930-1965). Santiago de Chile: Editorial Austral, 1965.

Ross, César. "Chile y Japón: El impacto del quiebre de la democracia en Chile, 1973", Atenea 492 (2005): 121-134.

“Auge y caída de Japón en Chile, 1897-1943”, Estudios Políticos 43 (2013): 156-179.

Sánchez, Freddy. "La relación de Latinoamérica y los Estados Unidos desde la visión de Salvador Allende Gossens", Cuadernos de Historia Contemporánea 25 (2003): 275288.

Sater, William F. Chile and the United States: Empires in conflict. Athens, Ga [u.a.]: University of Georgia Press, 1990.

Stallings, Barbara \& Székely, Gabriel. Japón, los Estados Unidos y América Latina: ¿hacia una relación trilateral en el hemisferio occidental?. 1a Edición. México, D.F.: Fondo de Cultura Económica, 1994.

Ulianova, Olga (Ed.), Redes políticas y militancias. Santiago de Chile: USACH-Ariadna editores, 2009.

UP. Programa básico de gobierno de la Unidad Popular: Centro de Estudios Bicentenario, 1969.

Wilhelmy, Manfred. "Chilean foreign policy: The Frei government, 1964-1970”. Tesis. Ph. D.. E.E.U.U.: Princeton University, 1976.

Fuentes:

Archivo Histórico del Ministerio de Relaciones Exteriores de Chile (AHMRREE), años 1971, 1972 y 1973: Notas, Oficios y Telex (Ordinarios y Reservados). 